\title{
Excitation and Reemission of Molecules near Realistic Plasmonic Nanostructures
}

\author{
Andreas M. Kern* and Olivier J. F. Martin* \\ Swiss Federal Institute of Technology Lausanne (EPFL), Nanophotonics and Metrology Laboratory, 1015 Lausanne, Switzerland
}

Supporting Information

\begin{abstract}
The enhancement of excitation and reemission of molecules in close proximity to plasmonic nanostructures is studied with special focus on the comparison between idealized and realistically shaped nanostructures. Numerical experiments show that for certain applications choosing a realistic geometry closely resembling the actual nanostructure is imperative, an idealized simulation geometry yielding significantly different results. Finally, a link between excitation and reemission processes is formed via the theory of optical reciprocity, allowing a transparent view of the electromagnetic processes involved in plasmon-enhanced fluorescence and Raman-scattering.
\end{abstract}

KEYWORDS: Surface integral equation, realistic nanoparticle, nanoantenna, near-field, SERS, fluorescence enhancement

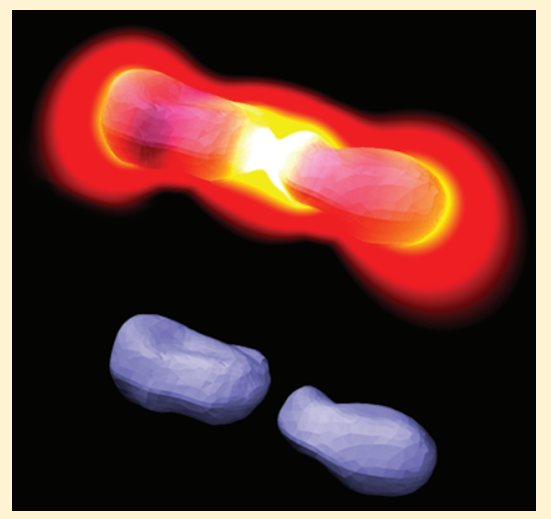

Tn the past decade, one important application of plasmonic nanostructures has been the exploitation of their localized field enhancement for amplifying fluorescence and Raman scattering. ${ }^{1-5}$ On the experimental side, astonishing single-molecule sensitivity has been demonstrated ${ }^{6-13}$ while large numbers of numerical investigations have been performed studying the effect of size and shape on the optical response, and thus the tunability of such structures. ${ }^{14-19}$ While the performed simulations studied many aspects of the particles' geometric configuration, for the most part they were restricted to idealized geometric primitives such as cylinders and prisms, which often bear only limited resemblance to realistic, fabricated structures. ${ }^{20}$ In addition, many reports on numerical studies show only the structures' farfield response, for example, the scattering or extinction cross sections. ${ }^{21,22}$ Fluorescence or Raman enhancements, however, depend on the near-field properties of the structure, which can differ quite drastically from observations in the far-field. In this study, we investigate the effect of some such simplifications often made in numerical analyses on the validity of their results, our main focus set on the difference between realistic and idealized objects.

When simulating nanophotonic structures, one can choose from a wide range of methods. Differential equation formulations such as the finite element method (FEM) ${ }^{23}$ or the finite difference time-domain (FDTD) method ${ }^{24}$ are flexible in their application but require absorbing boundary conditions for simulating isolated particles. Other specialized methods yield very accurate results, but only for particular geometries, for example, rigorous coupled-wave analysis (RCWA) for gratings and periodic geometries. $^{25}$ Integral equation (IE) methods have proved to be very useful in scattering analyses as their calculations fulfill the Sommerfeld radiation condition ${ }^{26}$ and thus intrinsically include the complete far-field information. In particular, surface integral equation (SIE) methods, also referred to as boundary element methods (BEM), have grown in popularity in optics ${ }^{27}$ as they unify a range of advantages otherwise not found in a single method. Unstructured meshes allow for efficient and accurate discretization while only the structures' surfaces have to be discretized, reducing the simulation complexity, especially for large structures. In addition, while all IE methods intrinsically supply farfield information, SIE methods in particular are able to compute the extreme near-field response of the simulated structures, making this approach ideal for the current study. For the presented simulations, we use an in-house SIE code that has been shown to yield accurate results in the near and far fields even in resonant plasmonic conditions. ${ }^{28}$

To explore the differences between realistic and idealized structures, two specific geometries were considered. The idealized case was represented by a regular, rectangular dipole nanoantenna as shown in Figure 1a. The antenna's arms have a $40 \times$ $40 \mathrm{~nm}^{2}$ cross section and a length of $98.5 \mathrm{~nm}$, edges rounded to a $5 \mathrm{~nm}$ radius, separated by a gap of $25 \mathrm{~nm}$ width. As a realistic structure, a nonregular dipole antenna based on a scanning electron microscope (SEM) image of a real fabricated antenna was derived, as shown in Figure $1 \mathrm{~b}$. The two antennae are about the same size and the nonregular case might easily represent the

Received: September 15, 2010

Revised: November 23, 2010

Published: January 4, 2011 

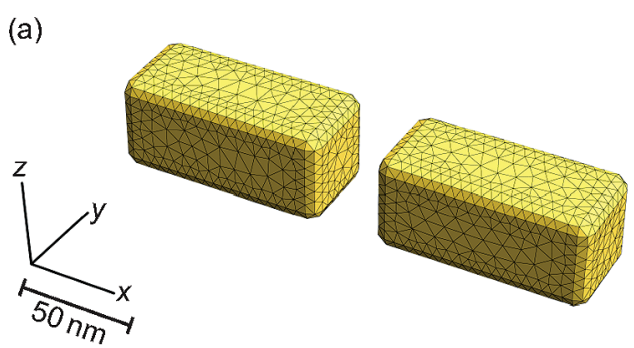

(b)

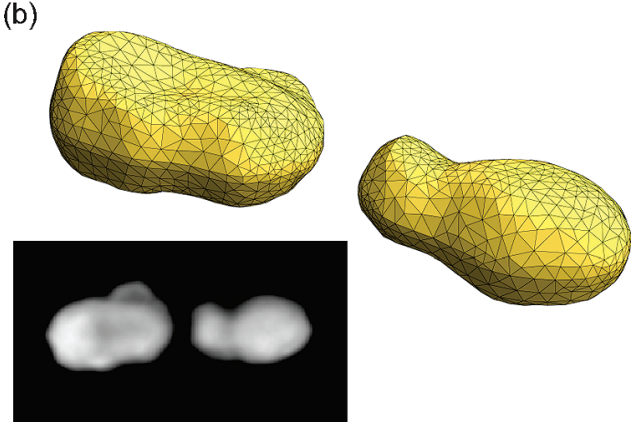

Figure 1. Studied geometries: (a) idealized rectangular nanoantenna and (b) realistic nanoantenna derived from SEM image (inset).

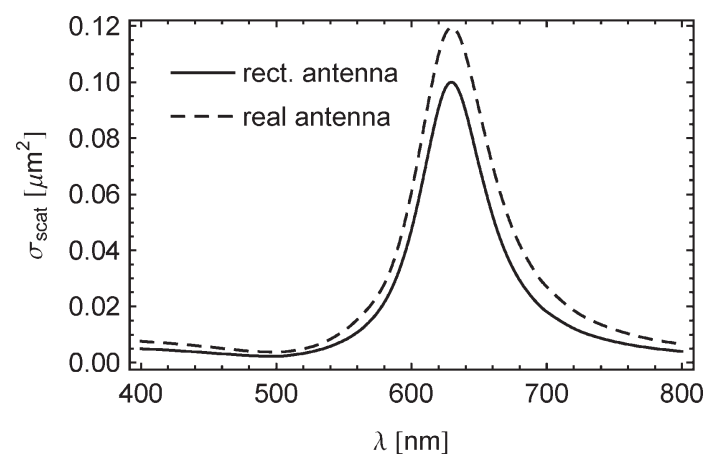

Figure 2. Scattering cross sections of idealized and realistic dipole antennae as shown in Figure 1.

fabricated result of the idealized design. Both antennae are modeled as gold, the dielectric constant taken from experimental data. ${ }^{29}$ As the nanostructures are modeled as regions of bulk metal, a possible crystallite substructure is not considered in this study. It might, however, be taken into account by using a modified dielectric function. ${ }^{30}$

While the geometries of the two cases differ substantially, an initial characterization might not be able to show the difference. Figure 2 shows the scattering cross sections of both antennae upon $x$-polarized plane wave excitation incident from the $+z$ direction. This demonstrates the behavior one might observe in a dark-field microscope measurement of the given antennae. While the absolute amplitudes of the resonance peaks are slightly different, the resonance wavelength of the two geometries are indistinguishable. A close look at the near-field distribution, however, makes clear that the antennae do not behave as similarly as the scattering cross section suggests. Figure 4 shows the intensity enhancement of the resonantly excited rectangular and realistic nanoantennae on a surface $1 \mathrm{~nm}$ outside the antennae's arms unwrapped using an equirectangular projection like a map

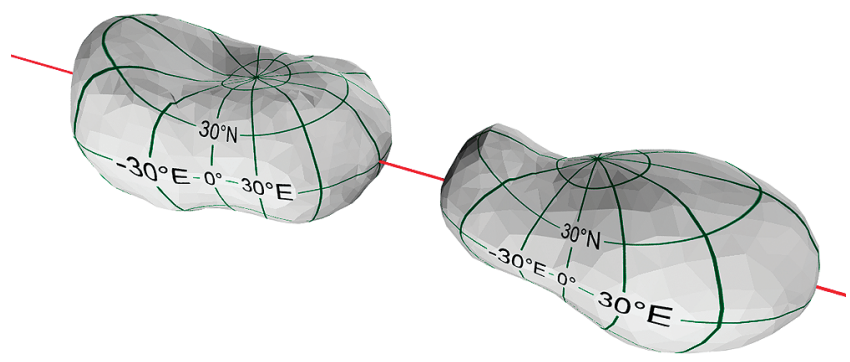

Figure 3. Coordinate system used for equirectangular projection of realistic nanoantenna. The antenna's axis corresponds to the $x$-axis and is shown by the red line.

of the globe, the antennae's axes intersecting the surfaces at $\left(0^{\circ} \mathrm{N}\right.$, $\left.-90^{\circ} \mathrm{E}\right)$ and $\left(0^{\circ} \mathrm{N}, 90^{\circ} \mathrm{E}\right)$, see Figure 3 . The resonance wavelength is chosen according to the maximum in Figure $2, \lambda_{\text {res }}=$ $630 \mathrm{~nm}$. Figure 4a shows the left and right arms of the idealized, rectangular antenna and Figure $4 \mathrm{~b}$ shows those of the realistic case. Clearly the field distribution is very different for both geometries, the rectangular antenna showing "hot spots" near its corners while the realistic antenna shows the highest field strength near the arms' centers. While in both cases the areas of highest field enhancement are located in the gap between the antennae's arms and at the arms' outside edges, the actual field distributions are indeed different and far from homogeneous as sometimes assumed. ${ }^{31}$ This is particularly important when considering plasmonic nanoantennae as a mediator for localized detection or trapping at the nanoscale. ${ }^{32,33}$

An interesting question is how close to the antenna one has to be for the difference in geometries to become noticeable. To this end, the intensity enhancement was mapped similarly to in Figure 4 but at distances of 1, 2, 5, and $10 \mathrm{~nm}$ from the antennae's left arms, shown in Figure 5. While the maps at $1 \mathrm{~nm}$ show a very different field distribution, the difference diminishes quickly with the maps at $10 \mathrm{~nm}$ appearing to be almost identical. When using plasmonic structures to enhance fluorescence or Raman scattering, the active particles are normally localized near the structure either deposited directly on its surface, separated by a dielectric spacer of few $\mathrm{nm}$ thickness or, if chemical selectivity is desired, via surface functionalization at distances on the order of $2-5 \mathrm{~nm}$. In any case, the distance to the plasmonic structure is very small and the active particles indeed experience a field distribution depending critically on the geometry and topology of the enhancing structures. Thus, for such cases, taking the actual geometry of a structure into account is imperative to achieve accurate simulation results.

At the same time, we can see that to accurately simulate the enhancement properties of plasmonic nanostructures the precise knowledge of the extreme near-field of such structures must be obtained. Of the methods mentioned in the introduction, most are capable of accurately determining the intermediate near-field of a given geometry, giving correct results down to a few nanometers above the structure's surface. At close proximities, however, many popular methods fail to accurately reproduce the field due to the used discretization. Volume integral equation (VIE) methods such as the discrete dipole approximation (DDA), for example, will show ripples caused by the discrete dipoles used to model the scatterer ${ }^{34,35}$ while the FDTD may introduce staircasing artifacts due to the structured grid required by the simulation technique. ${ }^{36}$ On the other hand, methods allowing for unstructured grids and continuous basis functions 

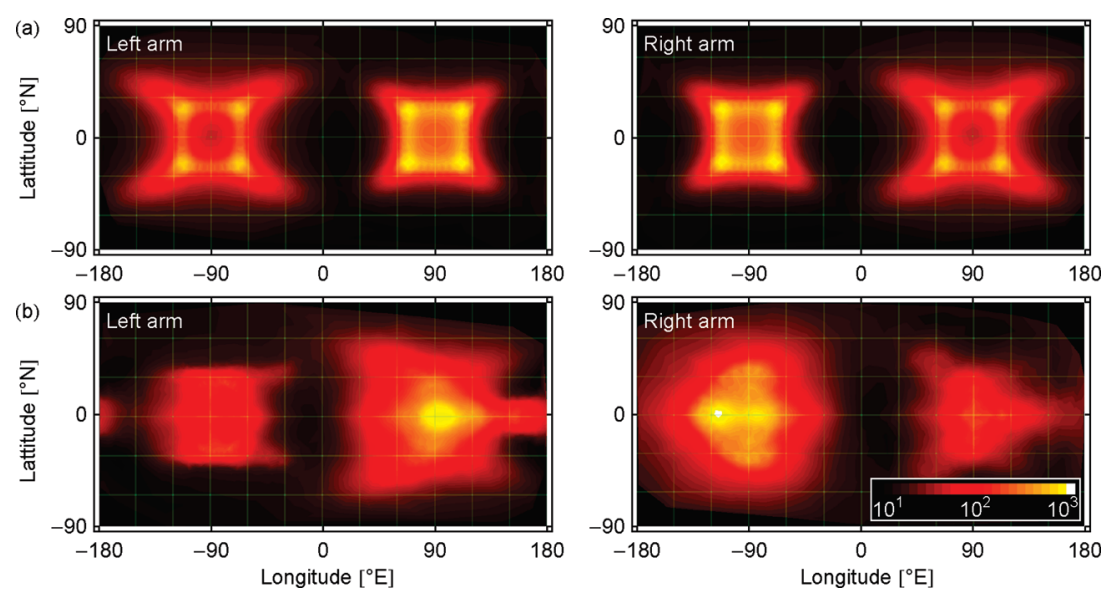

Figure 4. Intensity enhancement of a resonantly excited nanoantenna $\left(\lambda_{\text {res }}=630 \mathrm{~nm}\right)$ mapped on a surface $1 \mathrm{~nm}$ above the surface of the antennae. Map is unwrapped using an equirectangular projection (Figure 3).
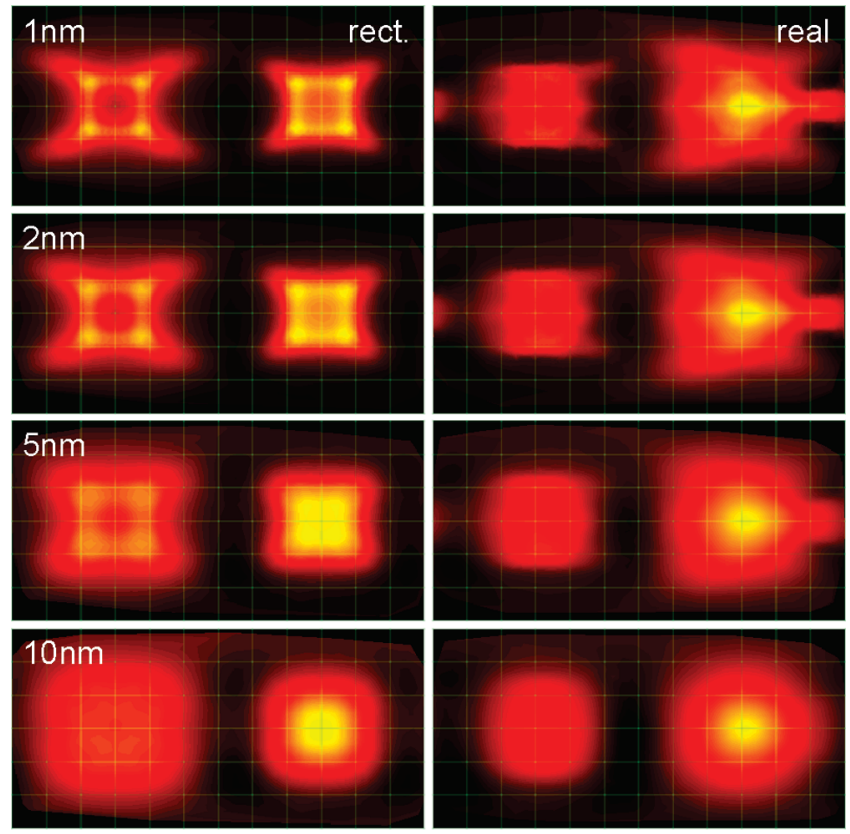

Figure 5. Unwrapped intensity maps at distances of 1, 2, 5, and $10 \mathrm{~nm}$ from surface of the antennae's left arms for resonantly excited rectangular and real antennae. Scale as in Figure 4.

such as the FEM or the SIE method are capable of delivering the required precision.

It should be noted that the electromagnetic simulations performed in this study do not take quantum mechanical effects into account, satisfying the classical Maxwell's equations only. Recently, fully quantum mechanical time-dependent density functional theory (TDDFD) has been applied in a fascinating investigation of the effects of electron spill-out on the tunability and field enhancement properties of small plasmonic nanostructures. ${ }^{37,38}$ In these studies, quantum effects were shown to have a noticeable effect on the field distribution around very small $(<10 \mathrm{~nm}$ in size) plasmonic particles. The effects, however, were limited to distances of less than $0.5 \mathrm{~nm}$ from the particles' surfaces. While the authors expect nonclassical behavior to extend to larger distances for larger particles, it remains unclear if effects will be noticeable at distances of $1-10 \mathrm{~nm}$ as studied here. In addition, if the quantum effects are indeed present even at these distances, the importance of the difference between realistic and idealized structures remains unchanged.

Besides concentrating incident light in "hot spots," plasmonic structures can increase the efficiency of fluorescence and Raman scattering by enhancing the radiation of the re-emitted light to the far-field where it can be detected. ${ }^{39-42}$ In some studies, this mechanism is not considered with the focus set only on the localized enhancement of the incident field. ${ }^{16}$ In others, it is taken into account but at the cost of a completely new set of calculations, as the chosen methods are not capable of simulating both effects. ${ }^{43}$ The SIE method used in this paper is capable of simulating a structure's response not only under plane wave illumination but also excited by a dipole source placed arbitrarily close to its surface. This makes it a suitable choice for simulating both the incident field enhancement and the radiation enhancement of light re-emitted from a fluorescent or Raman-active particle.

The solid lines in Figure 6 show the enhancement of the light radiated by a dipole source to the $+z$-direction, caused by the proximity to a rectangular $(a-c)$ or realistic $(d-f)$ plasmonic nanoantenna. The dipole source is located at three different points in the $x-z$-plane as indicated by the dots in the insets. Intensity enhancement is calculated for $x$-oriented (red lines) and $z$-oriented (green lines) dipole sources; the field is detected in the far-field at $(x, y, z)=\left(0,0,10^{6} \mathrm{~nm}\right)$ in $x$-polarization, parallel to the axis of the antennae. One can see that for a dipole located in the center of the gap $(\mathrm{a}, \mathrm{d})$ the intensity enhancement closely resembles the scattering cross section shown in Figure 2, the rectangular and realistic antennae displaying nearly the same behavior. The main difference, again, lies in the amplitude of the resonance peak, with the realistic case showing a slightly higher maximum enhancement (see Supporting Information, Figure S.1). Moving away from the center of the gap, however, the similarity between both geometries quickly vanishes. Not only do the proportions between $x$ - and $z$-polarizations differ but also the wavelengths at which the intensity is enhanced. The difference between polarizations is a direct effect of the shape of the scatterer: The coupling of a dipole to a metal surface strongly depends on the dipole's orientation relative to the surface. ${ }^{44}$ The spectral deviation between idealized and realistic antennae is the effect of the breaking of symmetry relative to the $x-y$-plane. In case of symmetry $(\mathrm{a}, \mathrm{d})$ the spectrum is predominantly defined by 

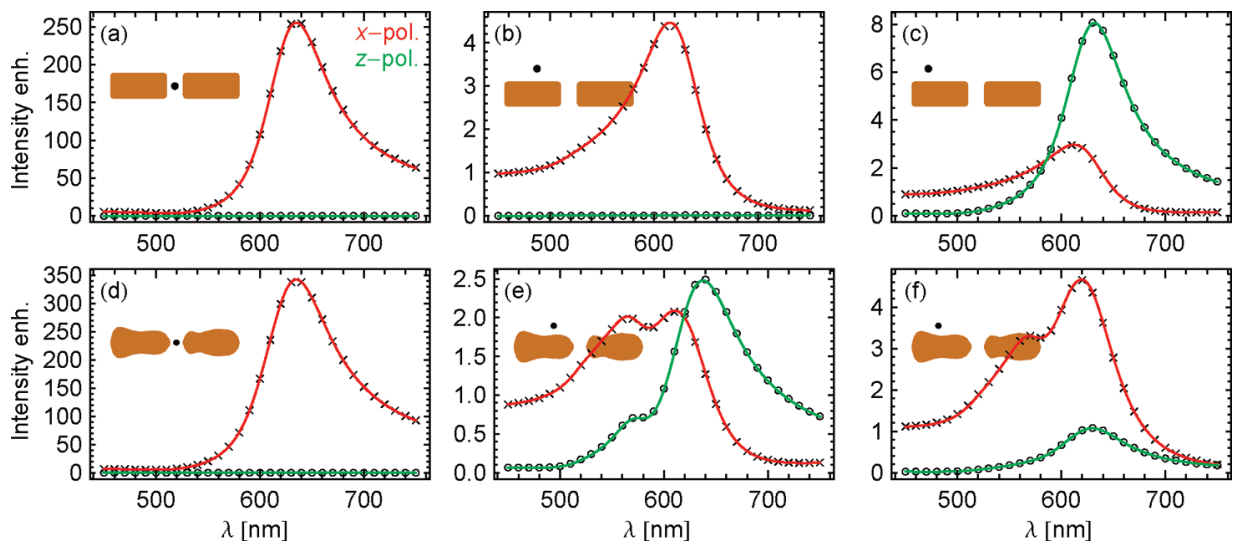

Figure 6. Solid lines show the enhancement of the intensity radiated to the far-field by a dipole source in the proximity of a rectangular (a-c) or realistic $(\mathrm{d}-\mathrm{f})$ nanoantenna. The source is located in the $x-z$-plane as indicated by the dots in the insets, oriented in $x$-direction (red curves) or $z$-direction (green curves) while far-field detection is at $(x, y, z)=\left(0,0,10^{6} \mathrm{~nm}\right)$ and in $x$-polarization. Symbols represent the reverse process with source and detection points exchanged: the $x$-polarized source is located at $(x, y, z)=\left(0,0,10^{6} \mathrm{~nm}\right)$ and detection is in the near field in $x$-polarization (crosses) and $z$-polarization (circles).

the antennae's geometry in $x$-direction (arm length and gap size), which is comparable for both geometries. Exciting the antennae away from their centers, other dimensions begin to play a role and the irregular shape of the realistic antenna causes its spectral response to be more complex than its idealized counterpart (see Supporting Information, Figure S.2).

While we have shown that the simulation routine used in this paper is capable of calculating the enhancement factors for both far- to near-field coupling and vice versa, this is not even necessary in many cases. The symbols in Figure 6 show the exact reverse process of the corresponding solid curves with the enhancement of a dipole source placed in the far-field and detected at different positions and polarizations in the near-field of the antennae. While it may at first seem remarkable that the solid curves and symbols show the exact same behavior, this can easily be explained using the theory of electromagnetic reciprocity, which allows the interchanging of electric current densities and the resulting electromagnetic fields. ${ }^{45,46}$ Reciprocity states that

$$
\mathbf{p}_{1} \cdot \mathbf{E}_{2}\left(\mathbf{r}_{1}\right)=\mathbf{p}_{2} \cdot \mathbf{E}_{1}\left(\mathbf{r}_{2}\right)
$$

where $\mathbf{p}_{1}, \mathbf{p}_{2}$ are two dipole sources at the positions $\mathbf{r}_{1}, \mathbf{r}_{2}$ and $\mathbf{E}_{1}(\mathbf{r}), \mathbf{E}_{2}(\mathbf{r})$ are the resulting electric fields in the given system. Denoting $\mathrm{p}_{1}$ as the distant dipole and $\mathrm{p}_{2}$ as the dipole in the nearfield of the structure, the polarization-dependent field enhancements invoked by the nanoantenna can be written as

$$
F_{12}=\frac{\mathbf{p}_{2} \cdot \mathbf{E}_{1}\left(\mathbf{r}_{2}\right)}{\mathbf{p}_{2} \cdot \mathbf{E}_{1}^{0}\left(\mathbf{r}_{2}\right)}, \quad F_{21}=\frac{\mathbf{p}_{1} \cdot \mathbf{E}_{2}\left(\mathbf{r}_{1}\right)}{\mathbf{p}_{1} \cdot \mathbf{E}_{2}^{0}\left(\mathbf{r}_{1}\right)}
$$

where $F_{12}$ and $F_{21}$ are the enhancements in the case of far-field and near-field dipole illumination, respectively, and $\mathrm{E}^{0}$ is the respective field without the antenna. Utilizing the transposition properties ${ }^{26}$ of the dyadic Green's function $\overline{\mathbf{G}}^{0}$ used to calculate $\mathbf{E}_{i}^{0}(\mathbf{r})=\overline{\mathbf{G}}^{0}\left(\mathbf{r}, \mathbf{r}_{i}\right) \cdot \mathbf{p}_{i}$,

$$
\begin{aligned}
\mathbf{p}_{2} \cdot \overline{\boldsymbol{G}}^{0}\left(\mathbf{r}_{2}, \mathbf{r}_{1}\right) \cdot \mathbf{p}_{1} & =\mathbf{p}_{1} \cdot \overline{\boldsymbol{G}}^{0}\left(\mathbf{r}_{2}, \mathbf{r}_{1}\right)^{\mathrm{T}} \cdot \mathbf{p}_{2} \\
& =\mathbf{p}_{1} \cdot \overline{\boldsymbol{G}}^{0}\left(\mathbf{r}_{1}, \mathbf{r}_{2}\right) \cdot \mathbf{p}_{2}
\end{aligned}
$$

it can easily be shown that $F_{12}=F_{21}$. From eq 2 one can see that the factors $F$ describe the effect of the antenna on the system's dyadic Green's function. ${ }^{47}$
This simple relation has significant meaning in the study of plasmonic structures as a means for emission enhancement. It states that the effect of a structure as an amplifier for the radiative decay of localized emitters, including polarization dependence, can be described by the field enhancement around the same structure upon far field illumination, obtainable in a single simulation. So simply repeating the simulation shown in Figure 4 for the shifted emission wavelength would result in a similar field distribution, this time showing areas in which a fluorescent or Raman-active molecule would efficiently couple to the far-field, the vectorial field direction representing the orientation of the corresponding dipole emitter. Only if the emission enhancement into a large solid angle is to be calculated, as when using a highnumerical aperture microscope objective, would a simulation with a near-field source be required, as the method above using reciprocity only considers emission in a single spatial direction, viz.: the relative position of the far-field illumination source.

Already the comparison between two structures performed in this study clearly demonstrates the difference between using a realistic geometrical model in optical simulations and using a simplified, idealized design. In some cases, such as when determining a structure's scattering properties, a simplified model is adequate. This is the case, for example, when studying the use of plasmonic scatterers to enhance the efficiency of solar cells ${ }^{48,49}$ or light-emitting diodes. ${ }^{50-52}$ When interested in the structure's near-field, however, such as when studying the use of plasmonic structures for fluorescence enhancement or optical trapping, the difference becomes substantial. Not only does the magnitude of the field enhancement depend on the structure's geometry, but so, too, does its distribution, dictating where the intensity will be maximal. If the materials to be exposed to light are widely dispersed over the whole area of the structure, the maximum field enhancement factor plays a dominant role, less its spatial distribution. In cases where localized detection is important, however, such as when combining fluorescence with optical trapping of single particles, ${ }^{33,53,54}$ knowledge of the field distribution is of eminent importance, as otherwise a particle may be localized in a position at which only low field enhancement occurs.

The second big difference between the optical properties of idealized and realistic structures is their response with respect to 
polarization and wavelength, as shown in Figure 6. Clearly, the two structures behave the same only in certain conditions, that is, regarding a particle in the center of the antennae's gaps, preserving symmetry in the system's geometry. If this symmetry is lifted, the similarity between idealized and realistic cases vanishes. Again, this difference is only relevant in certain applications. Considering the magnitude of the field enhancement shown in Figure 6, one can see that the symmetric cases $(\mathrm{a}, \mathrm{d})$ show by far the greatest values. Thus, if the active materials are randomly or broadly dispersed, the greatest contribution will come from these locations, and the precise geometry of the structure will play only a minor role on the system's response. If the location of the active particle is important, however, and it is placed at a position not preserving symmetry, the effect of the nanostructure's geometry will become important and its consideration in such simulations absolutely necessary.

In conclusion, we have compared the optical response of two plasmonic nanoantennae, one with an idealized geometry and the other realistically shaped. Both far-field and near-field response was investigated using a program based on the SIE method. While the far-field showed a similar response between the two geometries, the near-field properties of the two structures were distinctly different. In particular, the near-field distribution as well as the polarization and spectral behavior differed between the two, especially in cases showing low symmetry and thus encouraging the irregular shape of the realistic antenna to affect the optical response. Simulations were matched with possible applications, discriminating between those in which the exact geometry of the investigated structure is only of minor importance, and those where its consideration would be imperative. The former includes using structures purely as scatterers and cases where near-field enhancement need not be localized, whereas the latter describes cases where field enhancement is paired with particle localization and trapping.

\section{ASSOCIATED CONTENT}

S Supporting Information. The intensity enhancement in the midgap plane of the antennae was studied in greater detail. This material is available free of charge via the Internet at http:// pubs.acs.org.

\section{AUTHOR INFORMATION}

\section{Corresponding Author}

*E-mail: (A.M.K.) andreas.kern@epfl.ch; (O.J.F.M.) olivier.martin@ epfl.ch.

\section{ACKNOWLEDGMENT}

The authors gratefully acknowledge Christian Santschi and Weihua Zhang for fabrication and microscopy of plasmonic nanoantennae. This work was supported by the Swiss National Science Foundation (Grant 20021-116758) and the European Community's Seventh Framework Programme (FP7-ICT-2009-4, Grant agreement 248835).

\section{REFERENCES}

(1) Kelly, K. L.; Coronado, E.; Zhao, L. L.; Schatz, G. C. J. Phys. Chem. B 2003, 107, 668-677.

(2) Jackson, J. B.; Halas, N. J. Proc. Natl. Acad. Sci. U.S.A. 2004, 101, 17930-17935.

(3) Talley, C. E.; Jackson, J. B.; Oubre, C.; Grady, N. K.; Hollars, C. W.; Lane, S. M.; Huser, T. R.; Nordlander, P.; Halas, N. J. Nano Lett. 2005, 5, 1569-1574.
(4) Wei, H.; Hao, F.; Huang, Y.; Wang, W.; Nordlander, P.; Xu, H. Nano Lett. 2008, 8, 2497-2502.

(5) Li, J. F.; Huang, Y. F.; Ding, Y.; Yang, Z. L.; Li, S. B.; Zhou, X. S.; Fan, F. R.; Zhang, W.; Zhou, Z. Y.; Wu, D. Y.; Ren, B.; Wang, Z. L.; Tian, Z. Q. Nature 2010, 464, 392-395.

(6) Nie, S.; Emory, S. R. Science 1997, 275, 1102-1106.

(7) Nie, S.; Zare, R. N. Annu. Rev. Biophys. Biomol. Struct. 1997, 26, $567-596$.

(8) Kneipp, K.; Wang, Y.; Kneipp, H.; Perelman, L. T.; Itzkan, I.; Dasari, R. R.; Feld, M. S. Phys. Rev. Lett. 1997, 78, 1667-1670.

(9) Xie, X. S.; Trautman, J. K. Annu. Rev. Phys. Chem. 1998, 49, 441-480.

(10) Kneipp, K.; Kneipp, H.; Itzkan, I.; Dasari, R. R; Feld, M. S. Chem. Rev. 1999, 99, 2957-2976.

(11) Jiang, J.; Bosnick, K.; Maillard, M.; Brus, L. J. Phys. Chem. B 2003, 107, 9964-9972.

(12) Kühn, S.; Håkanson, U.; Rogobete, L.; Sandoghdar, V. Phys. Rev. Lett. 2006, 97, No. 017402.

(13) Dieringer, J. A.; Lettan, R. B.; Scheidt, K. A.; Van Duyne, R. P. J. Am. Chem. Soc. 2007, 129, 16249-16256.

(14) Nordlander, P.; Le, F. Appl. Phys. B 2006, 84, 35-41.

(15) Grzelczak, M.; Pérez-Juste, J.; Abajo, F. J. G. d.; Liz-Marza, L. M. J. Phys. Chem. C 2007, 111, 6183-6188.

(16) Fischer, H.; Martin, O. J. F. Opt. Express 2008, 16, 9144-9154.

(17) Myroshnychenko, V.; Rodriguez-Fernandez, J.; Pastoriza-Santos, I.; Funston, A. M.; Novo, C.; Mulvaney, P.; Liz-Marzan, L. M.; Abajo, F. J. G. d. Chem. Soc. Rev. 2008, 37, 1792-1805.

(18) Le, F.; Brandl, D. W.; Urzhumov, Y. A.; Wang, H.; Kundu, J.; Halas, N. J.; Aizpurua, J.; Nordlander, P. ACS Nano 2008, 2, 707-718.

(19) Koh, A. L.; Bao, K.; Khan, I.; Smith, W. E.; Kothleitner, G.; Nordlander, P.; Maier, S. A.; McComb, D. W. ACS Nano 2009, 3, 30153022.

(20) Barnes, W. L. J. Opt. A: Pure Appl. Opt. 2009, 11, 114002.

(21) Jensen, T. R.; Schatz, G. C.; Van Duyne, R. P. J. Phys. Chem. B 1999, 103, 2394-2401.

(22) Rodriguez-Fernandez, J.; Funston, A. M.; Perez-Juste, J.; Alvarez-Puebla, R. A.; Liz-Marzan, L. M.; Mulvaney, P. Phys. Chem. Chem. Phys. 2009, 11 (5909), 1463-9076.

(23) Monk, P. Finite Element Methods for Maxwell's Equations; Oxford University Press: Newark, 2003.

(24) Taflove, A.; Hagness, S. C. Computational Electrodynamics: The Finite-Difference Time-Domain Method, 2nd ed.; Artech House: Boston, 2000.

(25) Moharam, M. G.; Grann, E. B.; Pommet, D. A.; Gaylord, T. K. J. Opt. Soc. Am. A 1995, 12, 1068-1076.

(26) Chew, W. C. Waves and Fields in Inhomogeneous Media; Van Nostrand Reinhold, New York, 1990.

(27) Romero, I.; Aizpurua, J.; Bryant, G. W.; García de Abajo, F. J. Opt. Express 2006, 14, 9988-9999.

(28) Kern, A. M.; Martin, O. J. F. J. Opt. Soc. Am. A 2009, 26, $732-740$.

(29) Johnson, P. B.; Christy, R. W. Phys. Rev. B 1972, 6, 4370-4379.

(30) Sotelo, J.; Ederth, J.; Niklasson, G. Phys. Rev. B 2003, 67, 195106.

(31) Zhou, F.; Liu, Y.; Li, Z.-Y.; Xia, Y. Opt. Express 2010, 18, 1333713344 .

(32) Zhang, W.; Fischer, H.; Schmid, T.; Zenobi, R.; Martin, O. J. F. J. Phys. Chem. C 2009, 113, 14672-14675.

(33) Zhang, W.; Huang, L.; Santschi, C.; Martin, O. J. F. Nano Lett. 2010, 10, 1006-1011.

(34) Xiao, M. Opt. Commun. 1996, 132, 403-409.

(35) Wolf, E.; Foley, J. T. Opt. Lett. 1998, 23, 16-18.

(36) Cangellaris, A.; Wright, D. IEEE Trans. Antennas Propag. 1991, $39,1518-1525$.

(37) Zuloaga, J.; Prodan, E.; Nordlander, P. Nano Lett. 2009, 9, 887-891.

(38) Zuloaga, J.; Prodan, E.; Nordlander, P. ACS Nano 2010, 4, 5269-5276. 
(39) Moskovits, M. Rev. Mod. Phys. 1985, 57, 783-826.

(40) Blanco, L. A.; Garca de Abajo, F. J. Phys. Rev. B 2004, 69, 205414.

(41) Schatz, G.; Young, M.; Van Duyne, R. Surface-Enhanced Raman Scattering. In Topics in Applied Physics; Kneipp, K., Moskovits, M., Kneipp, H., Eds.; Springer: Berlin/Heidelberg, 2006; Vol. 103; pp $19-45$.

(42) Giannini, V.; Sánchez-Gil, J. A.; Muskens, O. L.; Rivas, J. G. J. Opt. Soc. Am. B 2009, 26, 1569-1577.

(43) Miao, X.; Brener, I.; Luk, T. S. J. Opt. Soc. Am. B 2010, 27, 15611570.

(44) Calander, N. Anal. Chem. 2004, 76, 2168-2173.

(45) Landau, L.; Lifchitz, E.; Pitaevskii, L. Electromagnetics of Continuous Media; Pergamon: Oxford, 1984.

(46) Ru, E. L.; Etchegoin, P. Chem. Phys. Lett. 2006, 423, 63-66.

(47) Martin, O. J. F.; Girard, C.; Dereux, A. Phys. Rev. Lett. 1995, 74, $526-529$.

(48) Westphalen, M.; Kreibig, U.; Rostalski, J.; Lüth, H.; Meissner, D. Sol. Energy Mater. Sol. Cells 2000, 61, 97-105.

(49) Catchpole, K. R.; Polman, A. Opt. Express 2008, 16, $21793-$ 21800 .

(50) Matterson, B. J.; Lupton, J. M.; Safonov, A. F.; Salt, M. G.; Barnes, W. L.; Samuel, I. D. W. Adv. Mater. 2001, 13, 123-127.

(51) Hobson, P.; Wedge, S.; Wasey, J.; Sage, I.; Barnes, W. Adv. Mater. 2002, 14, 1393-1396.

(52) Greiner, H.; Martin, O. J. F. Proc. SPIE Int. Soc. Opt. Eng. 2004, $5214,248-259$.

(53) Chaumet, P. C.; Rahmani, A.; Nieto-Vesperinas, M. Appl. Opt. 2006, 45, 5185-5190.

(54) Huang, L.; Maerkl, S. J.; Martin, O. J. Opt. Express 2009, 17, 6018-6024. 